\title{
Development of a Container Terminal Simulation Ontology
}

\author{
Ann-Katrin Lange ${ }^{1^{*}}$, Giovanni Pirovano ${ }^{2}$, Rosella Pozzi ${ }^{3}$, Tommaso Rossi $^{3}$
}

${ }^{1}$ Institute of Maritime Logistics of Hamburg University of Technology, Schwarzenbergstraße 95 D, 21073 Hamburg, Germany; * ann-kathrin.lange@tuhh.de

${ }^{2}$ Dept. of Management, Economics and Industrial Engineering of Politecnico di Milano University, Via Lambruschini 4/b, Milan, Italy

${ }^{3}$ Industrial Engineering School of LIUC - Cattaneo University, C.so Matteotti, 22 - 21053 Castellanza, Italy

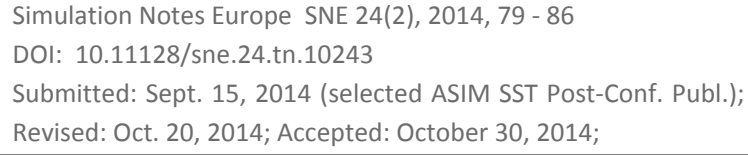

Submitted: Sept. 15, 2014 (selected ASIM SST Post-Conf. Publ.); Revised: Oct. 20, 2014; Accepted: October 30, 2014;

Abstract. This article introduces a simulation ontology to support terminal planers, operator and managers in the design and management of seaport container terminals. Due to the increasing requirements of shipping companies regarding efficiency, quality and price for the handling processes at container terminals, the use of integrated approaches for improving the performance has grown significantly. Simulation, which has proven highly beneficial in production and logistics, represents an adequate tool to deal with complex systems like container terminals. However, building simulation models requires much time and simulation software know-how. To counteract this effect, this article presents a simulation ontology of seaport container terminals, which supports the user in building specific simulation models.

Since the simulation model is automatically created through the ontology framework, neither the personnel skills nor the time available to build the simulation model represent significant hurdles. Furthermore, the proposed ontology can dramatically reduce the time required to test a specific configuration of a container terminal and/or a particular management policy. The ontology framework consists of a user interface with database, where the user can specify elements and their parameters, an atom library representing all elements of the system and software application, which is used to automatically build the simulation model.

\section{Introduction}

Around 80 per cent of global merchandise trade by volume were transported by water and therefore handled in ports in 2012 [1]. This fact highlights the further growing strategic economic importance of seaborne transport. Combined with low and at the same time volatile freight rates for maritime transport, still remaining after the economic crisis in 2008, and rising bunker prices, this development stimulates the ongoing increase of ship dimensions. This derives a special significance for container vessels, which transport around 52 per cent of global seaborne trade in terms of value [2].

The size of the largest container vessels has more than doubled from around 8,000 twenty-foot equivalent units (TEU) in 2004 to over 18,000 TEU in 2013. Without optimization of the container handling, this would lead to much longer berthing times for unloading and loading the vessels and, as a consequence, to sharp rising port charges. But due to the weak bargaining position of container terminals in relation to shipping companies [3], the terminal operators are pressed to optimize their efficiency and productivity while keeping up low prices. Therefore, all operations and services at the container terminal have to be evaluated and, if necessary, redesigned and adjusted with high investments to meet the stringent demands of a higher turnover in short time windows and higher quality [4].

Seaport container terminals can be considered, in term of material flow, as open systems with two interfaces to other linked systems [5]. 
One of the interfaces is the quayside, where container ships are assigned to a specific berth and discharged and charged by a set number of ship-to-shore cranes. The other interface is the landside with the unloading and loading of trucks and trains, which can be carried out be different kinds of internal equipment, e.g. rubbertired-gantry cranes or straddle carriers. The transport of containers from the interfaces to the stocking yard or vice versa is carried out by horizontal transport means, which may differ depending on the required task.

As a result, there is a big variance in the used internal equipment in all areas of the terminal, depending on many different factors, e.g. the stocking systems in the container yard, the average and maximum size of the landing container vessel, the labour costs in the area, the available space in the port, security requirements and the demanded productivity $[4 ; 5 ; 6]$.

Recent developments are e.g. the increasing automation of handling processes to reduce labour costs and to optimise quality and using advanced spreaders to lift multiple containers for enhancing the productivity as well as lowering costs.

To plan, analyse, manage and optimise the complex system of a container terminal, it is no longer sufficient to rely on the knowledge of singular experts or on the problem solving competence of departments in specific, isolated areas. Therefore, integrated approaches for improving the performance of container terminals have been developed. Apart from analytical approaches, there is a focus on simulation based approaches [4], which have proven highly beneficial as decision support systems in production and logistics in general [7] and for container terminals in particular $[8 ; 9 ; 10]$.

For newly planned container terminals, simulation models can provide a preview of the expected overall performance and support the identification of problems before implementing the system. For already existing container terminals, simulation can help to identify bottle necks and optimisation potentials in the current situation and compare them to alternative operation approaches, which can be tested easily and without risks.

This article introduces a methodology to support decisions of seaport container terminal planners, operators and managers concerning the terminal layout, equipment and operations by developing a simulation ontology.
This simulation ontology overcomes the limitations of usual simulation models whose building requires much time and simulation software know-how of the user. Instead it enables the user to quickly build specific simulation models by simply entering all relevant characteristics of the seaport container terminal and its equipment in a user friendly interface.

The article is structured as follows. Section 1 provides information on the state of research about simulation models of container terminals on the one hand and about simulation ontologies on the other hand. Section 2 presents the proposed methodology for the simulation ontology framework for container terminals by explaining the architecture, the atoms library and the software application. Finally, some concluding remarks and suggestions for future research are described in Section 3.

\section{Background}

Seaport container terminals are complex systems because of several reasons. First of all, there exists a wide variety of organisational forms in regard to terminal operations and used equipment. Many factors have to be considered: How is the layout of the terminal? Which modes of transportation are connected to the terminal? What kind of vessels can be discharged and charged (e.g. maximum size)? Is there a freight station? What kind of equipment is used? Are some processes automated? Are there special areas for storing empty containers, dangerous goods or reefer containers?

Second, after evaluating the organisational form of the terminal, many decisional variables have to be considered, e.g. the number of every kind of equipment and its capacity and the speed of horizontal and vertical transport. In addition, many of these variables may be linked and, as a consequence, influence each other.

Third, static constraints have to be considered as well as dynamic ones. Static constraints are e.g. the number of bays available for the landing of container vessels or the direction of roads on the terminal. Dynamic constraints are for example the work schedules of the staff, the repair schedules of the equipment and the arrival times of ships, trains and trucks at the container terminal.

Fourth, extreme weather conditions and failures of the equipment represent sources of uncertainty, which have to be taken into account. 
Because of the complexity, modelling a whole container terminal analytically has proven a challenge $[10 ; 11]$ and the popularity of simulation models for this task has grown significantly [e.g. 6;12;13]. Many of the simulation models focus on one specific area of operation of a container terminal e.g. automated container yard blocks or automated container terminals [14;15], management of berth crane operations $[16 ; 17]$ or the analysis of horizontal transport means [18]. Furthermore, there are some simulation models representing one whole, specific container terminal $[11 ; 19]$.

Although simulation models are important parts of industrial system analysis and control system design, the simulation design issue, the fact that manual simulation design is known as time consuming and error-prone work, has not yet been solved [20].

As a way to overcome such an issue, literature has turned to ontologies, which are a way of formalizing knowledge in a machine-understandable form. In detail, ontologies can be defined as a collection of the kinds of entities that exist in a domain (an identified system), their properties, and the relationships that can hold between them [21]. Ontologies also deal with concepts as ontology classes and individuals, i.e. instances of ontology classes.

Novák and Šindelář formalize knowledge on largescale industrial systems and use a semi-automated semantic engine that assembles the simulation model, introducing a significant impact of using ontologybased methods on simulation model design phase [20].

Miller et al. develop an example of an ontology for discrete-event modelling, which is a very general domain, identifying the concepts that are most relevant for the discrete-event modelling domain, the relationships between them, the overall architecture, and some of the technical steps involved in creating, deploying and using such an ontology [22].

The present project aims at developing the ontology framework of container terminal simulation models. The individual simulation model of a system associated to the container terminal, which the ontology refers to, is given by a particular instance of the ontology class [20;21;22], similarly to the individual simulation model which is a particular instance of the ontology it refers to [20;23;24]. This instance is automatically obtained based on user-specified input data.

\section{The Proposed Methodology}

The container terminal simulation ontology framework presented in this paper is based on the simulation software package 'Enterprise Dynamics' (ED) of Incontrol Simulation Solutions.

In the following sections, the overall architecture of the simulation ontology (Section 2.1), the relevant objects (2.2) and the software application (Section 2.3) are presented.

\subsection{Ontology architecture}

The ontology allows the container terminal manager both to define by means of classes the topology, the resources and the characteristics of the real container terminal under study and to automatically build the corresponding simulation model. By experimenting on such a model, the container terminal manager can verify in advance the performance of the terminal and makes decisions to improve such performance.

The ontology framework involves (see Figure 1): (1) a user interface with database; (2) an ad hoc objects library; (3) a software application. Through the user interface, the container terminal manager specifies both elements and elements' characteristics (the values of the parameters the elements are characterised by) of the container terminal: the values manually entered by the terminal manager and the ones calculated by the same interface are recorded into the database.

The ad hoc library contains both ED atoms and specifically conceived atoms that represent the building blocks of a container terminal: each atom is described by data, represented by the atom attributes, and behaviour, modelled by 4Dscript code (4DScript is the programming language ED is based on). The atom attributes are the parameters that characterise the corresponding container terminal element, while the behaviour is the simulation sub-model, which represents how the corresponding element behaves.

Finally, the software application allows the simulation model to be automatically built. From the database, the application reads the elements of the container terminal and, for each of them, it:

- (1) selects the corresponding atom from the ad hoc library; 
- (2) selects from the database the values to be assigned to each atom attribute;

- (3) makes the assignments;

- (4) inserts into the simulation environment the (parameterised) atom.

Once the software application has completed the abovementioned steps for all the elements of the container terminal, the simulation model is built and the terminal manager can experiment on it.

The atoms library and software application are presented in sections 2.2 and 2.3, respectively. There is no section devoted to either the database or the user interface: actually the database consists of a standard ED table atom, while the interface is given by mere dataentry masks, which allow to specify the values of the container terminal elements parameters, which are the mirror image of the objects data.

\subsection{Atoms library}

Two different classes of objects belong to the ad hoc library: (1) the roads, which allow to create the network where the transport means characterising the container terminal move; (2) the resources, which perform the activities within the container terminal: gate, service line, rail crane, active transportation equipment (i.e. straddle carrier, reach-stacker, container lift truck), passive transportation equipment (automated guided vehicles, tractor and trailer, multi-trailer), stacking yard and portal crane.
Trucks, trains, ships and containers are not considered as objects since they are the entities which flow along the simulation models which in turn can be automatically built by the simulation ontology framework.

The objects are described by data and behaviour (as pointed out in Section 2.1). As for the data, the attributes related to the atoms of the roads class are: (1) the couple of nodes linked by the road; (2) the parameters required to set possible constraints (direction, number of driving lines, traffic rule and speed factor).

The attributes related to the atoms of the resources class basically quantify the terminal resources occupancy (storage capacity, cranes rate, inter-arrival traffic time at piers, etc.).

As for the behaviours, they are the simulation submodels which represent how the container terminal elements interact with each other and with the entities represented by trucks, trains, ships and containers. To provide an example, Table 1 presents the data and behaviour (i.e. the attributes and the simulation submodel) of the object (i.e. atom) 'active transportation equipment' (in particular, due to the functioning of ED, the sub-model is represented by means of state machine diagrams and attributed Petri nets).

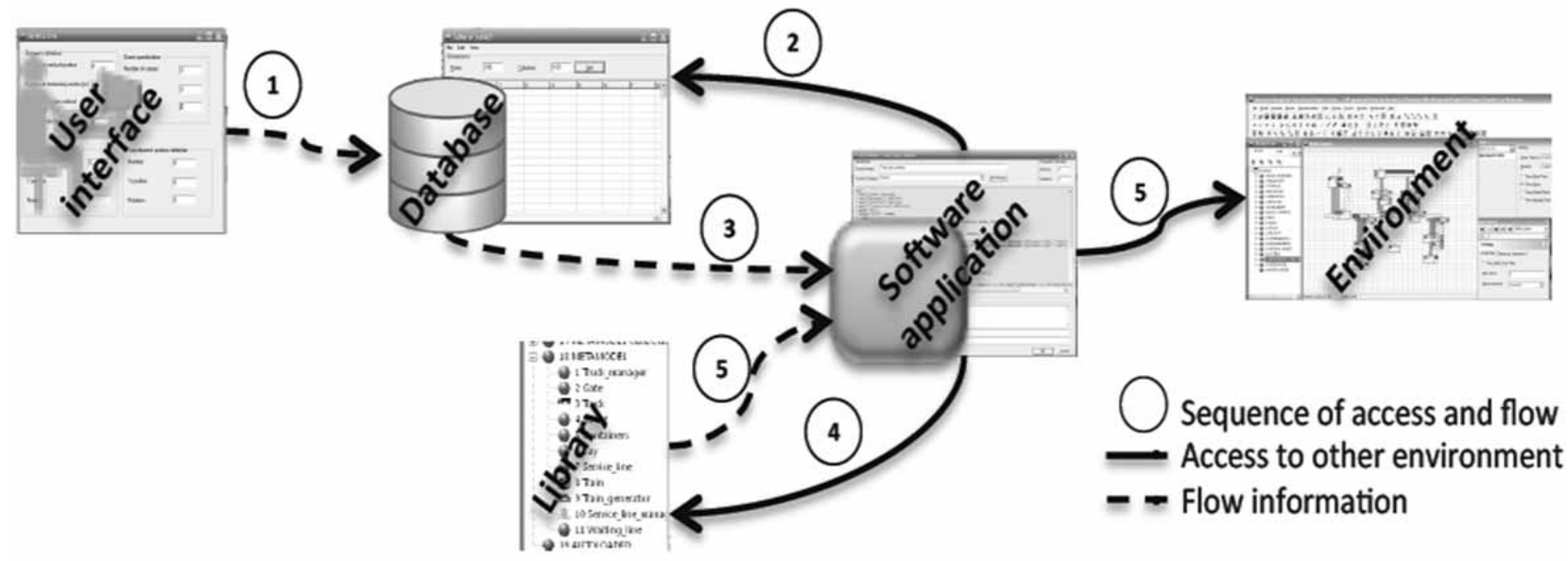

Figure 1. Ontology architecture. 


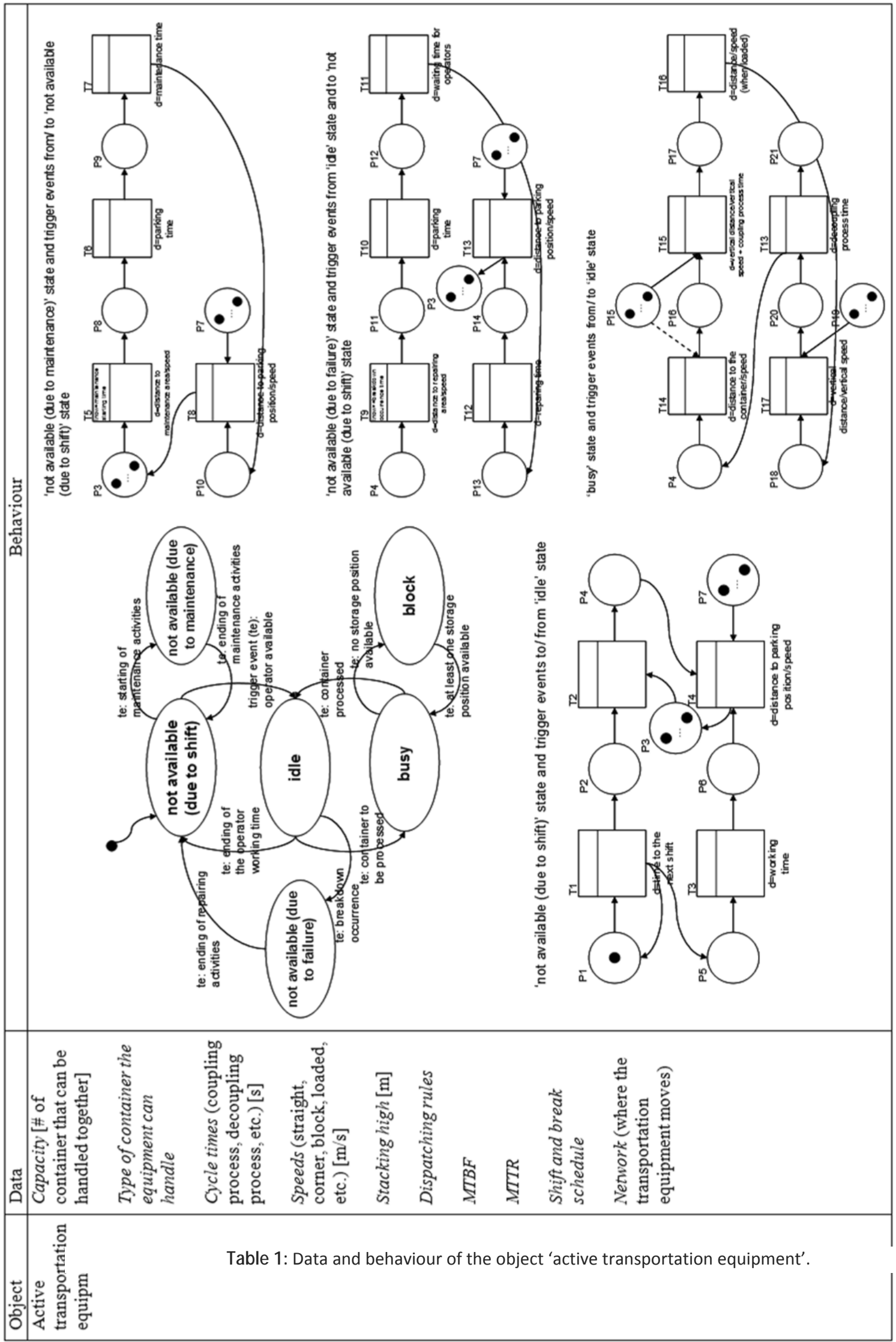




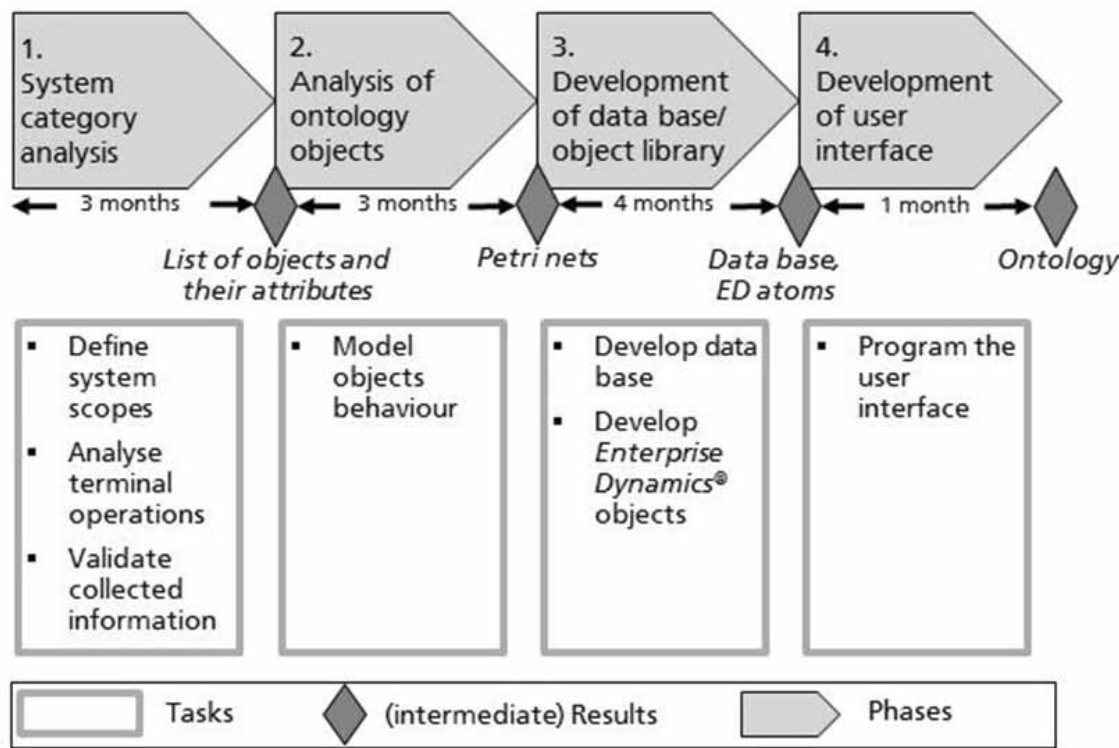

Then the software application chooses the road atom from the ad hoc library, inserts it into the simulation environment and assigns to the attributes of the atom a alue according to the previously red parameters.

At that time the second subprocedure stops, since the network is completely modelled into the simulation environment, the simulation model of the container terminal under study is ready to be used.

Figure 2: Project phases for container terminal ontology framework development

\subsection{Software application}

The software application is divided in two subprocedures: the first one inserts into the simulation environment the atoms representing the objects belonging to the resources class; the second creates the network by inserting a road atom between all the couples of resource atoms, which represent elements that are included into the real container terminal network.

The first sub-procedure starts by accessing the database, i.e. the table atom, and by reading all the parameters related to the first object belonging to the resources class, which must be created. Then the software application accesses the ad hoc atom library and inserts into the simulation environment the corresponding atom. After that, the application assigns the previously red values to the specific atom.

This sub-procedure is repeated for all the resources objects defined by the user. When all the resources objects have been created, the first sub-procedure ends and the second one starts, in order to create the network. In particular the sub-procedure reads again from the table atom the parameters (allowed speed, number of driving lines, etc.) that characterize the link between the first couple of resources objects.

\section{Conclusion and Outlook}

This article is focused on the development of an ontology to support the design and the management policies definition of a seaport container terminal.

The reason to address this problem is given by the fact that, despite simulation is considered one of the most promising tools to support the design and to manage container terminals; its use in real-life contexts is limited by the high requirements for development time and simulation know-how.

To address the problem a joint project is carried on by the Institute of Maritime Logistics of Hamburg University of Technology - MLS (Hamburg, Germany) and Cattaneo University - LIUC and Politecnico di Milano University. The project is structured in four phases (see Figure 2). Currently, phase 3 is in progress.

The first phase has dealt with the deep analysis of the category of systems which the simulation ontology refers to, i.e. container terminals. A set of questions have been asked to practitioners to obtain necessary information. In addition, inspections to exemplar maritime container terminals in Hamburg and Bremerhaven have been performed. Literature has been used to gather further input from other container terminals and to consider all well-known established and planned types of terminal operation systems. 
The output of the first phase has been identified by a list of all the objects the simulation ontology must include and the attributes characterizing each object (see paragraph 3.1. For an example of attributes, see column 'Data' of Table 1).

In the second phase, the logical model representing the behaviour of each listed object has been developed. In order to facilitate the development process and to ensure the completeness of the logical model a state machine diagram has been constructed for every object. This diagram displays all the states that the object can visit, the trigger events for changing into another state and the action performed before and after the state change. Based on these state machine diagrams the Petri nets formalism has been used to display the internal behaviour during the different states and the behaviour during a state change (for an example, see table 1). The developed state machine diagrams and Petri nets identify the output of the phase.

The third phase of the project, which is in progress, consists of the development of the database and of the ad hoc object library by means of ED. So far, the database has been developed by means of the ED atom 'Table'; the ad hoc atoms representing the objects 'road', 'service line', 'rail crane' 'stacking yard', 'tractor and trailer' have been developed in 4DScript; a beta version of the software application to automatically build the simulation model has been coded in 4DScript.

In the last phase of the project a user interface will be developed by means of the ED application 'GUI builder'. This interface will allow the user to define the elements composing the container terminal which shall be simulated as well the container terminal topology. Furthermore, it will allow the user to easily enter the necessary values into the database to define the attributes of each system element.

The user interface will be built so as that, on the one hand, the user can decide for many of the attributes if he/she wants to enter values or use the deposited ones and, on the other hand, the input of data can as well be done by loading complex lists, e.g. ship arrival times, as by filling out dialog windows. The completed container terminal ontology framework will identify the output of the last phase.
The main strengths of the proposed simulation ontology can be summarised as follows: first, it represents a specifically conceived decision support tool for solving an optimisation problem under several constraints, uncertainty and many interdependent variables as the design or the definition of management policies of a container terminal is. Second, the proposed ontology allows to take into account some dynamic features of the terminal system unmanageable by manual calculations (e.g. the arrival times of ships, trains and trucks at the terminal).

Finally, the time required to test a specific configuration of the system is dramatically reduced: in a few minutes the user can specify the elements the system is composed of as well as the elements' characteristics through the user interface; the software application builds the corresponding simulation model, which can be run, depending on the simulation length and on the hardware, in a few seconds or in a few minutes, on behalf of the decision-making process speed.

The future of the research line outlined here is oriented towards proving the effectiveness of the proposed simulation ontology framework by using it to estimate the performance of an existing container terminal system: for this reason, a study of the La Spezia container terminal has been already planned.

\section{References}

[1] UNCTAD Secretariat. Review of Maritime Transport. United Nations Publication, Switzerland, 2013.

[2] World Shipping Council. Value of world seaborne trade. See http://www.worldshipping.org/ (accessed 10 July 2014), 2014.

[3] T.-F. Wang and K. Cullinane. The efficiency of european container terminals and implications for supply chain management. Maritime Econ Logistics 8, p. 82-99, 2006.

[4] R. Stahlbock and S. Voß. Operations research at container terminals: a literature update. OR Spectrum 30, p.1-52, 2008.

[5] D. Steenken, S. Voß and R. Stahlbock. Container terminal operations and operations research - a classification and literature review. OR Spectrum 26, p.3-49, 2004.

[6] P. Ghanbari. Containerterminal-Logistik. VDM Verlag Dr. Müller, Germany, 2007.

[7] L. März and G. Weigert. Simulationsgestützte Optimierung. In: Simulation und Optimierung in Produktion und Logistik, Editor: L. März, W. Krug, O. Rose und G. Weigert, Springer-Verlag Berlin Heidelberg, Germany, p. 3-12, 2011. 
[8] R. Gibson, B. Carpenter and S. Seeburger. A flexible port traffic planning model. Proceedings of the 1992 Winter Simulation Conference, in USA, p. 1296-1306, 1992.

[9] P.-H. Koh, J. L. K. Goh, H.-S. Ng and H.-C. Ng. Using simulation to preview plans of a container port operations. Proceedings of the 1994 Winter Simulation Conference, in USA, p. 1109-1115, 1994.

[10] Y. Merkuryev, J. Tolujew, E. Blümel, L. Novitsky, E. Ginters, E. Viktorova, G. Merkuryeva, J. Pronins. A modelling and simulation methodology for managing the Riga harbour container terminal. Simulation 71, p. 8495, 1998.

[11] W. Y. Yun and Y. S. Choi. A simulation model for container-terminal operation analysis using an objectoriented approach. International Journal of Production Economics 59, p. 221-230, 1999.

[12] L. M. Gambardella, A. E. Rizzoli and M. Zaffalon. Simulation and planning of an intermodal container terminal. Simulation 71(2): p. 107-116, 1998.

[13] T. Franz. Marktbasiertes ContainerterminalManagement - Grundlagen, Mechanismen und verteilte Simulation. VDM Verlag Dr. Müller, Germany, 2008.

[14] N. Kemme. Effects of storage block layout and automated yard crane systems on the performance of seaport container terminals. OR Spectrum 3, p. 563-591, 2012.

[15] P. Canonaco, P. Legato, R. Mazza and R. Musmanno. A queuing network model for the management of berth crane operations. Computers \& Operations Research 8, p. 2432-2446, 2008.

[16] C.-I. Liu, H. Jula and P. A. Ioannou. Design, Simulation, and Evaluation of Automated Container Terminals. IEEE Transactions on intelliegent transportation systems 3 no. 1, p. 12-26, 2002.

[17] J. Dai, W. Lin, R. Moorthy and C.-P. Teo. Berth allocation planning optimization in container terminal. Working paper, Georgia Institute of Technology, Atlanta; National University of Singapore, 2004.
[18] M.B. Duinkerken, R. Dekker, S.T. Kurstjens, J.A. Ottjes and N.P. Dellaert. Comparing transportation systems for inter-terminal transport at the Maasvlakte container terminals. OR Spectrum 28, p. 469-493, 2006.

[19] A.A. Shabayek and W.W. Yeung. A simulation for the Kwai Chung container terminal in Hong Kong. European Journal of Operational Research 140, p. 1-11, 2002.

[20] P. Novák and R. Šindelár. Applications of ontologies for assembling simulation models of industrial systems. On the Move to Meaningful Internet Systems: OTM 2011 Workshops. Springer Berlin Heidelberg. p. 148-157, 2011.

[21] P. Benjamin, M. Patki and R. Mayer, Using ontologies for simulation modeling. In: Proceedings of the 38th conference on Winter simulation. Winter Simulation Conference, p. 1151-1159, 2006.

[22] J. A. Miller, G. T. Baramidze, A. P. Sheth, G. A. Silver and P. A. Fishwick, Ontologies for modeling and simulation: An initial framework. Computer Science Department, University of Georgia, Athens, GA, 30602, 2004.

[23] R. Cigolini and T. Rossi. Sizing off-shore transshipment systems in dry-bulk transportation. Production Planning \& Control 21, p. 508-522, 2010.

[24] R. Cigolini, M. Pero and T. Rossi. An object-oriented simulation meta-model to analyse supply chain performance. International Journal of Production Research 49, p. 5917-5941, 2011.

[25] V. Boschian, M. Dotoli, M.P. Fanti, G. Iacobellis and W. Ukovich. A Metamodeling Approach to the Management of Intermodel Transportation Networks. IEEE Transactions on Automation Science and Engineering 8 no. 3, p. 457-469, 2011 